\section{Selection of protein epitopes for antibody production}

\author{
Mats Lindskog, Johan Rockberg, Mathias Uhlén, and Fredrik Sterky \\ Royal Institute of Technology, Stockholm, Sweden
}

BioTechniques 38:723-727 (May 2005)

Protein functional analysis in the post-genomic era is a huge task that has to be approached by different methods in parallel. The use of protein-specific antibodies in conjunction with tissue microarrays has proven to be one important technology. In this study, we present a strategy for the optimized design of protein subfragments for subsequent antibody production. The fragments are selected based on a principle of lowest sequence similarity to other human proteins, optimally to generate antibodies with high selectivity. Furthermore, the fragments should have properties optimized for efficient protein production in Escherichia coli. The strategy has been implemented in Bishop, which is a Java-based software enabling the high-throughput production of protein fragments. Bishop allows for the avoidance of certain restriction enzyme sites, transmembrane regions, and signal peptides. A Basic Local Alignment Search Tool (BLAST) scanning procedure permits the selection of fragments of a selected size with a minimal sequence similarity to other proteins. The software and the strategy were evaluated on a human test data set and verified to fulfill the requested criteria.

\section{INTRODUCTION}

A variety of technologies for studies of proteins on a proteomic scale are now available. The principal for analysis as well as the characteristics of the results differ dramatically. Much effort is being put into protein expression analysis by different separation techniques coupled to mass spectrometry $(1,2)$. Twohybrid screens (3) are used for studies of protein-protein interactions for functional characterization of proteins, and approaches for large-scale determination of protein structures have been developed (4). Another principally different approach is epitope tagging of proteins (5) for subcellular localization using monoclonal antibodies.

Recently, an approach to apply localization studies of human proteins in cells and tissues was presented (www. hpr.se). This antibody proteomics technology involves the large-scale production of monospecific polyclonal antibodies for the cellular and subcellular localization of human proteins in tissue sections and cell cultures (6). By using tissue arrays (7), proteins can be localized in a large number of tissues, cell cultures, and disease states. Images of the tissues are stored in a protein atlas, which offers a powerful tool for the functional determination of proteins. An important part of the antibody proteomics technology is to design subfragments of proteins, which will be amplified by reverse transcription PCR (RT-PCR) and cloned into expression vectors. These subfragments, hereafter called protein epitope signature tags (PrESTs), are optimized for production in Escherichia coli and are expected to represent unique protein epitopes.

The scope of this work was to implement a strategy for the optimal design of PrESTs and to develop userfriendly software (Bishop), allowing high-throughput production of the protein fragments. The strategy was evaluated on a human test data set. We also present results from a study examing the potential of doing experiments on mouse using antibodies derived from human-mouse orthologs.

\section{MATERIALS AND METHODS}

The strategy for the PrEST design is based on a set of rules that were developed for optimal protein production and purification as well as for ensuring a good immune response. The first rule was to avoid transmembrane regions. It has been reported that these regions are difficult to express in E. coli, and severe problems also occur in the refolding and purification process (8). In addition, transmembrane regions are not easily accessible by antibodies in immunolocalization studies. The second rule was to avoid signal peptides, since they are cleaved off during translocation and will therefore be unsuitable as epitopes. The third rule was to design a protein fragment of suitable size, small enough for easy handling by PCR and cloning and large enough to provide conformational epitopes. The optimal size is usually 100-150 amino acids, and for shorter gene products, the complete protein would be selected. The fourth rule was to make the amino acid sequence of the fragments as unique as possible (compared to other human proteins) to avoid cross-reactivity by the generated antibodies.

The software Bishop was developed under Linux using Java. The BioJava API (www.biojava.org) was used for sequence handling and visualization. The relational database PostgreSQL (9) was used for storage of input data and results. As seen below, several Linux programs were called for specific analyses by the main program. A demo of the software and additional information can be obtained online at biobase.biotech.kth.se/bishop.

\section{PrEST Selection Steps}

Bishop enables the user to perform a number of analyses of the selected sequence. When the correct parameters are set, the analyses are performed automatically or by manual execution of the steps below.

Identification of restriction enzyme sites. The user can select any restriction enzyme to be displayed. It is important to avoid the design of PrESTs in regions containing restriction enzymes used in subsequent cloning.

Prediction of signal peptides and transmembrane regions. Transmembrane regions are predicted by 
launching TMHMM software (10), and signal peptides are determined using SignalP (11). Both of these programs run under Linux, and the results are integrated and visualized in Bishop.

Scanning for unique regions. One of the most important criteria for the PrEST design is to find fragments that have the lowest sequence similarity as possible to other human proteins. To find such regions, a scanning procedure based on BLASTP (12) was developed. A sliding window of fixed size along the protein is compared to all other proteins in the Ensembl database (www.ensembl.org). The score and E-value for the strongest sequence similarity at each step is then recorded (Figure 1). The window size can be set to any value, and the window step is one residue between each BLASTP. Before the scan, there is an option to remove sequences with more than $95 \%$ sequence identity from the Basic Local Alignment Search Tool (BLAST) database. This will ensure an informative scan curve and indicate that special consideration has to be taken regarding the possible role of the removed proteins.

PrEST selection. In general, the program suggests two PrESTs (Figure 1). These are nonoverlapping fragments with the lowest BLASTP scores that lack transmembrane regions, restriction sites, and signal peptides and have a sequence similarity to other human proteins lower than a threshold (default $60 \%$ identical amino acid residues). If the user is not satisfied with the selected region, or if all suggestions failed, any region can be selected manually for further processing.

Primer design. To facilitate amplification and cloning of the designed fragments, a primer design module was included in Bishop. The user defines the desired melting temperature $\left(\mathrm{T}_{\mathrm{m}}\right)$ and the length of the primers. The $\mathrm{T}_{\mathrm{m}}$ is calculated from the simple formula: $\mathrm{T}_{\mathrm{m}}=(4 \times[\mathrm{G}+\mathrm{C}])+(2 \times[\mathrm{A}+\mathrm{T}])$ (13). In addition, the primers must end with a $G$ or a $C$ due to their stronger binding, and stretches of more than three (default) identical nucleotides are not permitted.

\section{Evaluation of Design Principles}

In order to investigate the impact of this set of rules and principles on real data, a number of analyses were performed. The 682 proteins from chromosome 22 in Ensembl (version 11.31.1) (14) were used as a test data set. For each protein, sequence similarity with all other proteins in Ensembl was determined by BLASTP. In the cases where subsequences within the data set were found, the longest one was selected. Furthermore, proteins with splice variants were removed from the data set, and a minimum of 250 amino acids was required. A total of 220 proteins were selected using these filters.

Bishop was then used to generate one PrEST fragment for each of the 220 peptides. Three different window sizes were used during the BLASTP scan: 50,125 , or 250 amino acids in length. Transmembrane regions, restriction sites, and signal peptides were avoided according to the set of rules and principles. Suggested PrESTs with a higher similarity than $60 \%$ to any other protein in the human data set

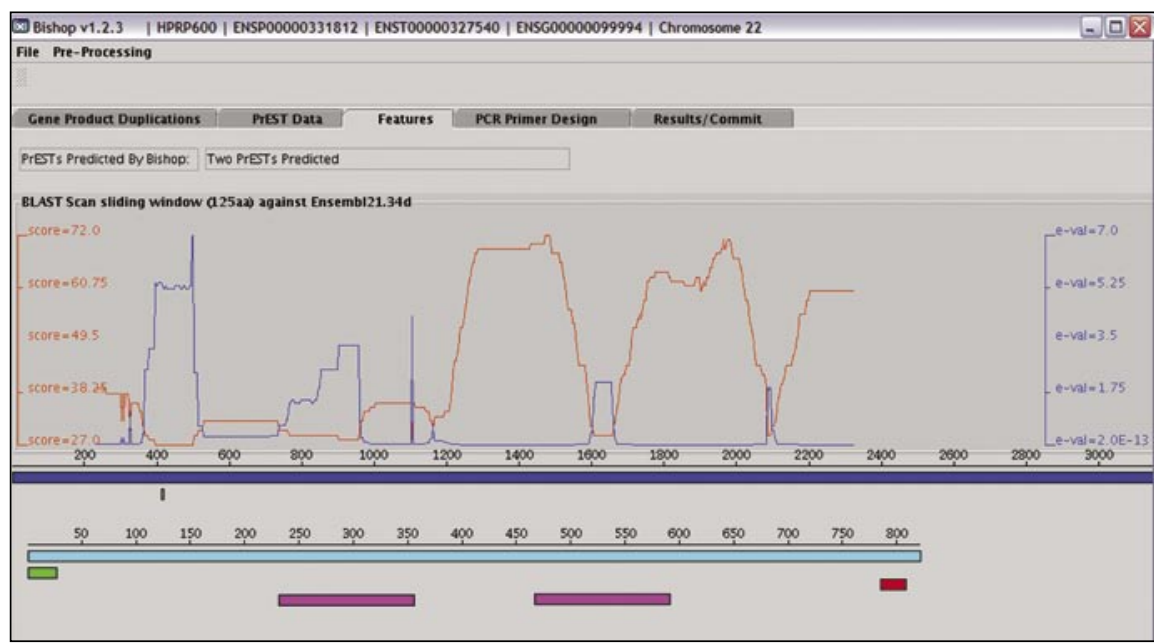

Figure 1. Screenshot from Bishop showing the Feature tab where results from the BLASTP scan are visualized. Gene/protein features are displayed in the lower panel: transcript sequence (dark blue), restriction site (orange), protein sequence (light blue), signal peptide (green), transmembrane region (red), and protein epitope signature tags (PrEST) sequence (magenta).

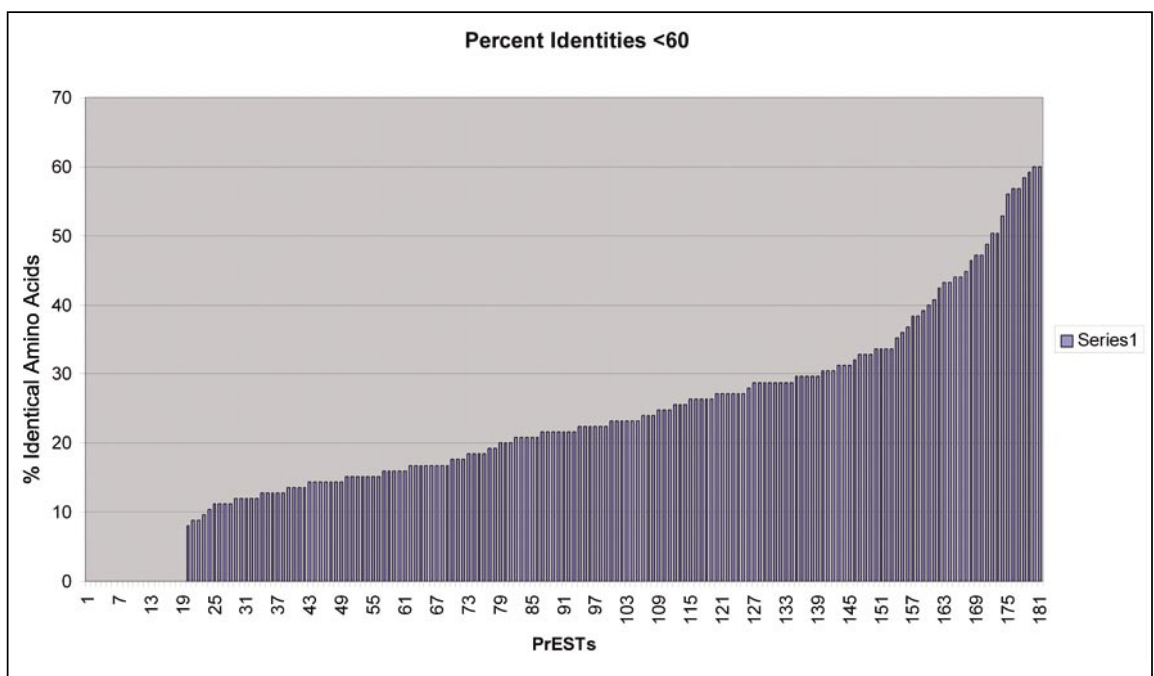

Figure 2. The proportion of identical amino acids for the successfully designed protein epitope signature tags (PrESTs; 125 amino acids) as compared to the best hit to any of the available proteins in Ensembl. Potential PrESTs with identities over $60 \%$ were discarded due to the risk of production of nonspecific antibodies. 
were rejected (Figure 2). The suggested PrESTs were compared to a random data set to evaluate if the program generated fragments that on average contained a lower number of conserved protein domains. The random data set was generated by randomly selecting a fragment for each protein, which gave a total of 220 PrESTs. This procedure was done for all three PrEST lengths and iterated 100 times. Finally, the PrESTs were analyzed by BLASTP against the conserved domain database (CDD; v.1.62) (15) at the National Center for Biotechnology Information (NCBI), and the number of hits was registered.

The PrEST fragments were further evaluated by analyzing their 3-dimensional (3-D) structures. For the 220 proteins on chromosome 22,8 protein structures were available from the Protein Data Bank (16) for genes where a PrEST had been successfully designed. The amino acid sequences corresponding to the PrESTs were marked in the 3-D structures of the protein (Figure 3) using Vector NTI Suite 8 (www.informaxinc.com). In order to evaluate the potential of a correctly folded PrEST, the C- and $\mathrm{N}$-terminals of the protein were also colored according to the different window sizes.

Antibodies against human genes are obviously of interest for use in other organisms. Such approaches are especially attractive in mouse. To evaluate the potential of human antibodies being functional in mouse, a comparison of all PrEST sequences to mouse orthologs (Ensembl v.11.31.1) for each gene was performed. The BLASTP algorithm was used for determining the sequence identities on different levels (Figure 4).

\section{RESULTS AND DISCUSSION}

The developed software Bishop is a selection and visualization software that makes use of the publicly available complete human genome sequence and allows the user to study different entities of the protein using publicly available programs, as well as determining the uniqueness of the subfragment using sequence similarity searches. Based on the generated information, a candidate PrEST is selected, and primers are automatically generated for amplification. The PrESTs are designed to contain unique epitopes that are present in the native protein and suitable for triggering the generation of antibodies of high selectivity. If the design principles described are not applicable (e.g., for short proteins, splice variants, proteins with several transmembrane regions, very similar proteins, etc.), the software allows for manual selections of epitopes according to other strategies.

The strategy and software were evaluated using a cleaned data set from Ensembl containing 220 proteins, all at least 250 amino acids long. PrEST fragments were generated for the three different window sizes. For some proteins, Bishop failed to select any PrEST for the requested window size. The failures are due to insufficient space left for design after the blocking of restriction sites, signal peptides, or transmembrane regions. Peptides were also failed if the sequence similarity of potential PrESTs never came below the threshold 60\% (Figure 2). For the window of 50 amino acids, the number of failed peptides was 20 ; for the window of 125 amino acids, the number of failed peptides was 39 ; and for the window of 250 amino acids, the number of failed peptides was 56 .

The main principle of Bishop is to design short fragments with as little similarity to other proteins as possible. The number of conserved domains in the PrESTs should therefore be lower than in randomly selected fragments. This hypothesis was tested by BLASTP searches against the CDD database, both on the PrESTs and also on the generated random data set. Null distributions were then generated from the randomly designed fragments. In order to determine significance levels, the fifth percentiles for each of the three randomized data sets were calculated. They were 528 for a length of 50 amino acids, 1634 for a length of 125 amino acids, and 1723

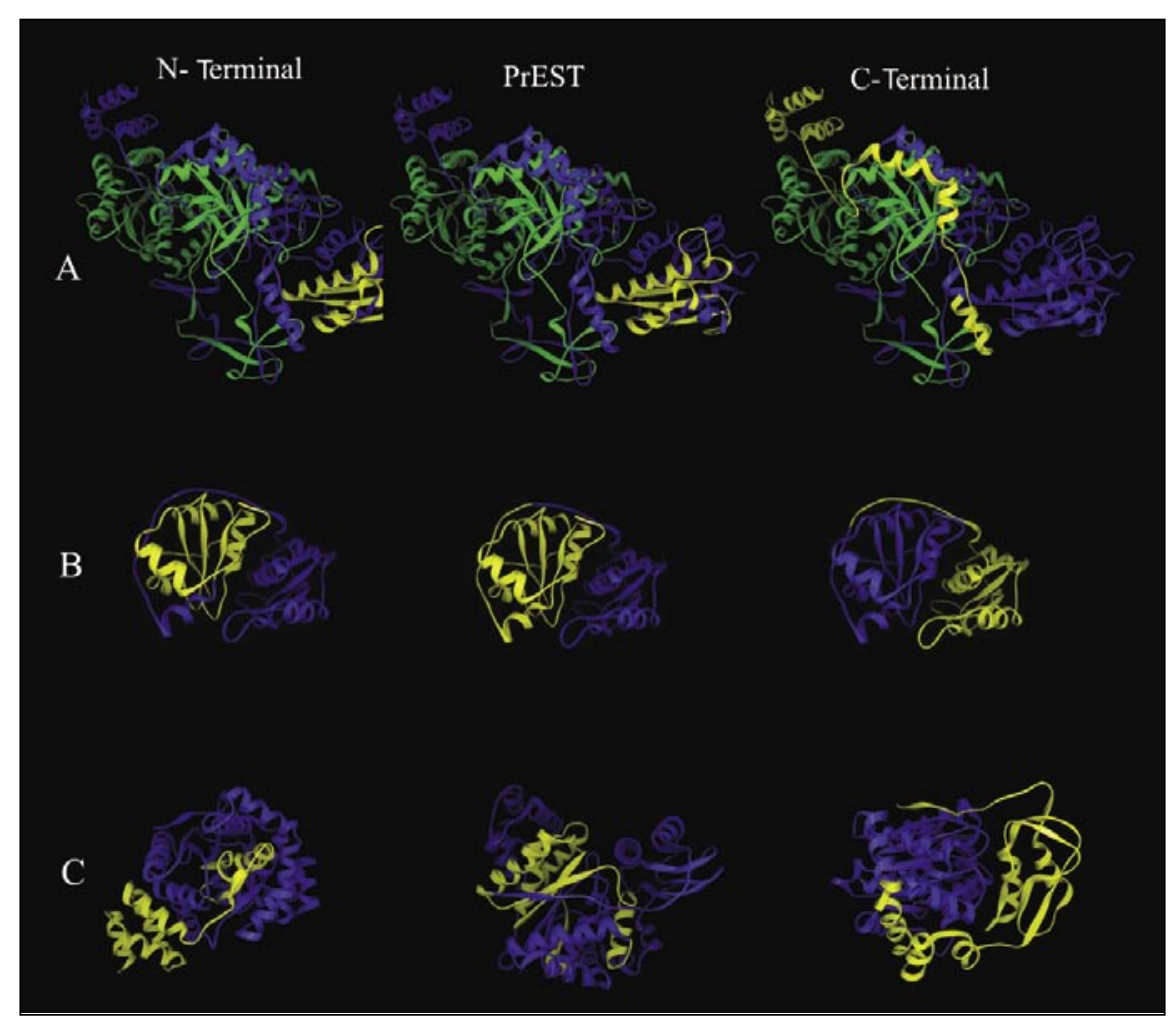

Figure 3. 3-Dimensional (3-D) structures of proteins corresponding to genes on chromosome 22: (A) 10RB, (B) 1UOU, and (C) 1JEQ. Fragments of 125 amino acids are colored in yellow on the original structure (blue). Green color indicates a protein dimer. The fragments have been colored according to three different principles: C-terminal, N-terminal, or lowest similarity protein epitope signature tags (PrEST; i.e., Bishop). 


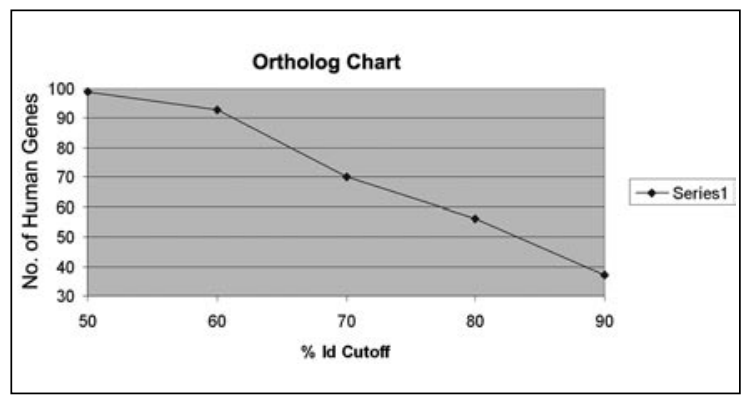

Figure 4. The number of protein epitope signature tags (PrESTs) designed on human genes that have a mouse ortholog at different amino acid sequence similarity cutoffs. The graph represents a PrEST length of 125 amino acids, but the results were very similar for 50 and 250 amino acids.

for a length of 250 amino acids. The results for the predicted PrESTs were 479 domains in 200 predicted PrESTs for a length of 50 amino acids, 1522 domains in 181 PrESTs for a length of 125 amino acids, and 1594 domains in 164 PrESTs for a length of 250 amino acids. Consideration has been taken to account for the fact that some proteins failed before the PrEST design, and the values above are weighted. The generated PrESTs were confirmed to contain fewer conserved domains than what was expected to occur by chance at the $5 \%$ level.

To obtain antibodies recognizing the native protein, it is important that the cloned and expressed PrESTs are forming conformational epitopes correctly representing the folded parts of the native protein. The PrESTs designed by the lowest similarity principle of Bishop show a variation in the potential of forming folded proteins (Figure 3). However, as suggested by the known 3-D structures, the PrEST designed by the software package seems to have a relatively good potential to display some conformational epitopes, as indicated by the inclusion of some complete secondary structures and the relative compactness of the PrEST structures. Although, this is by no means proof for the recombinantly expressed protein fragments forming secondary structures resembling the native protein, the folding potential of the Bishopgenerated protein fragments seems to have a similar potential forming native secondary structures as the fragments of similar lengths designed as $\mathrm{N}$ - or $\mathrm{C}$-terminals of the protein. $\mathrm{N}$ - or Cterminal fragments are commonly used when recombinant protein fragments are being expressed for the generation of epitopes for antibody production (6). The PrEST size also seems to be large enough to provide surface exposure for some residues on the native protein, which is of importance for antibody recognition of the target protein.

The similarity to known Mus musculus orthologous genes was investigated. According to Ensembl, 160 of the 220 human genes had known mouse orthologous. The predicted PrESTs were compared to the mouse data by BLAST at several levels of sequence similarity (Figure 4). By looking at how many of the human genes that had a PrEST designed with a certain similarity to at least one of the respective mouse orthologs, we were able to get an impression of the possibilities to use the generated antibodies for the mouse genes. For the 125 amino acid PrESTs, 23 of 181 PrESTs had a mouse ortholog with above $90 \%$ sequence similarity. For more than 50\% similarity, 99 of the 181 PrESTs had a mouse ortholog. The patterns were very similar for PrEST lengths of 50 and 250 amino acids.

In conclusion, in an effort to select optimal subfragments of human proteins for subsequent antibody production, we describe a design strategy and a software package named Bishop. The strategy is based on the identification of subfragments with minimal similarity to any other human protein. In order to facilitate cloning of the fragments, restriction sites are avoided. Furthermore, transmembrane regions are not allowed, since such fragments are hard to produce in E. coli. Finally, signal peptides are avoided, since they may result in antibodies with incorrect specificity. The Bishop software contains functions to deal with each of these issues in a userfriendly way. The software automatically suggests the PrESTs based on the design principles, but any sequence can be selected manually if desired. The approach of taking a transcript sequence through several analysis steps based on tabs in the user interface makes it easy to add new functionality to the design process.

By applying the strategy to a test set from chromosome 22, it was shown that the PrESTs contained significantly lower numbers of conserved domains than random PrESTs. This indicates that Bishop fulfills the desired function in finding the most unique parts of the proteins. By analyzing the few 3-D structures available for the test data, we could conclude that the fragments designed based on low-similarity had a potential on the same level as other strategies to form secondary structures similar to that of the native protein, which is important for the formation of conformational epitopes.

In addition, the strategy is at present applied in the generation of polyclonal antibodies (www.hpr. se). Polyclonals are advantageous as compared to monoclonal antibodies 
to overcome some limitations with the PrEST strategy. The PrESTs might have conformational differences to the native protein, and the produced PrEST proteins will not be glycosylated. In both of these cases, polyclonal antibodies are more likely to be functional due to the recognition of multiple epitopes.

Finally, many of the short PrESTs proved to be similar in sequence to the corresponding mouse orthologs. The antibodies produced by this concept should therefore be possible to apply on mouse tissues and retain their specificity to the target protein. This will be important for economical reasons as well as for research. The generated information is also important for the potential ability to distinguish between human and mouse orthologs.

\section{ACKNOWLEDGEMENTS}

We are thankful to Per Kraulis, Per Unneberg, Tyler Rimstad, Kristina Bergström, and Cristina Al-Khalili Szigyarto for contributing to this work by creative discussions. This work was supported by grants from the Knut and Alice Wallenberg (KAW) Foundation.

\section{COMPETING INTERESTS STATEMENT}

The authors declare no competing interests.

\section{REFERENCES}

1.Patterson, S.D. and R. Aebersold. 1995. Mass spectrometric approaches for the identification of gel-separated proteins. Electrophoresis 16:1791-1814.

2.Washburn, M.P., D. Wolters, and J.R. Yates III. 2001. Large-scale analysis of the yeast proteome by multidimensional protein identification technology. Nat. Biotechnol. 19:242247.

3.Fields, S. and O. Song. 1989. A novel genetic system to detect protein-protein interactions. Nature 340:245-246.

4.Hammarstrom, M., N. Hellgren, S. van Den Berg, H. Berglund, and T. Hard. 2002. Rapid screening for improved solubility of small human proteins produced as fusion proteins in Escherichia coli. Protein Sci. 11:313-321.

5.Kumar, A., S. Agarwal, J.A. Heyman, S. Matson, M. Heidtman, S. Piccirillo, L. Umansky, A. Drawid, et al. 2002. Subcellu- lar localization of the yeast proteome. Genes Dev. 16:707-719.

6.Agaton, C., J. Galli, I. Hoiden Guthenberg, L. Janzon, M. Hansson, A. Asplund, E. Brundell, S. Lindberg, I. Ruthberg, et al. 2003. Affinity proteomics for systematic protein profiling of chromosome 21 gene products in human tissues. Mol. Cell Proteomics 2:405-414.

7.Kononen, J., L. Bubendorf, A. Kallioniemi, M. Barlund, P. Schraml, S. Leighton, J. Torhorst, M.J. Mihatsch, et al. 1998. Tissue microarrays for high-throughput molecular profiling of tumor specimens. Nat. Med. 4:844-847.

8. Miroux, B. and J.E. Walker. 1996. Over-production of proteins in Escherichia coli: mutant hosts that allow synthesis of some membrane proteins and globular proteins at high levels. J. Mol. Biol. 260:289-298.

9.Momjan, B. 2000. PostgreSQL: Introduction and Concepts. Pearson Education. Reading, MA.

10.Krogh, A., B. Larsson, G. von Heijne, and E.L. Sonnhammer. 2001. Predicting transmembrane protein topology with a hidden Markov model: application to complete genomes. J. Mol. Biol. 305:567-580.

11.Nielsen, H., J. Engelbrecht, S. Brunak, and G. von Heijne. 1997. Identification of prokaryotic and eukaryotic signal peptides and prediction of their cleavage sites. Protein Eng. 10:1-6.

12.Altschul, S.F., W. Gish, W. Miller, E.W. Myers, and D.J. Lipman. 1990. Basic local alignment search tool. J. Mol. Biol. 215:403410 .

13.McConlogue, L., M.A. Brow, and M.A. Innis. 1988. Structure-independent DNA amplification by PCR using 7-deaza-2'-deoxyguanosine. Nucleic Acids Res. 16:9869.

14.Hubbard, T., D. Barker, E. Birney, G. Cameron, Y. Chen, L. Clark, T. Cox, J. Cuff, et al. 2002. The Ensembl genome database project. Nucleic Acids Res. 30:38-41.

15.Marchler-Bauer, A., A.R. Panchenko, B.A. Shoemaker, P.A. Thiessen, L.Y. Geer, and S.H. Bryant. 2002. CDD: a database of conserved domain alignments with links to domain three-dimensional structure. Nucleic Acids Res. 30:281-283.

16.Berman, H.M., J. Westbrook, Z. Feng, G. Gilliland, T.N. Bhat, H. Weissig, I.N. Shindyalov, and P.E. Bourne. 2000. The Protein Data Bank. Nucleic Acids Res. 28:235-242.

Received 13 October 2004; accepted 1 December 2004.

Address correspondence to Fredrik Sterky, Department of Biotechnology, KTH, AlbaNova University Center, SE-10691 Stockholm, Sweden. e-mail: sterky@kth.se 